EDITOR'S PAGE

\section{Refining and defining public health}

In this issue, Auger and Raynault (page 350) bring to our attention the potential of a 'Balanced Scorecard' to convey a persuasive message about health inequalities to a variety of audiences. This approach represents the next and necessary iteration of population health needs assessment, augmented by more deliberate consultation and greater awareness of its utility for advocacy and resource reallocation. Other articles in this issue invite consideration of indicators to extend the reach and role of public health.

For example, Schellenberg and colleagues (page 369) share a telling study of the gaps between public knowledge, behaviour and recommended public health practice. As shown by Yiannakoulias and colleagues (page 374), community factors are powerful predictors of population health. Sensitive, well-conducted studies of the health of vulnerable populations also feature in this issue. Dallaire and colleagues (page 362) reveal the impact of specific childhood infections upon isolated Inuit communities. Electronic health records were used by Podymow et al. to determine specific healthrelated episodes among homeless men in Ottawa (page 379). By comparing data from two provinces, Fischer and colleagues (page 384) display trends in premature mortality from drug-related overdoses. An illuminating study by Greaves et al. (page 388) carefully portrays unmet needs of marginalized women. Importantly, Sloka and colleagues show the population impact of folic acid supplementation programs in Newfoundland and Labrador (page 393). Children at risk of obesity are the subject of Galloway's article (page 353).

This issue also presents constructive critique of principles and practice in public health. Kenny and colleagues (page 402) argue the need for a strong ethical foundation for public health. Wenkui and colleagues (page 398) share their multi-layer framework to maximize response to public health emergencies in Guangxi, a province of almost 50 million people in China. Definitions of health and disability reflect much deeper assumptions and priorities for public health as discussed by Wolbring (page 405). Muldoon and colleagues provide a contemporary critique of primary care and primary health care (page 409) while Knaak (page 412) poses important questions about breastfeeding discourse. O'Reilly and colleagues shed light on the process of interdisciplinary research (page 415).

Once read, please return again to contemplate our cover. Imagine a 'Balanced Scorecard' that uses accurate and comprehensive data to create a new imperative for public health action.

Jeanette Ward, MBBS, MHPEd, PhD, FAFPHM

Interim Scientific Editor, CJPH
LE MOT DE LA RÉDACTION

\section{Peaufiner et définir la santé publique}

Dans ce numéro, Auger et Raynault (page 350) nous font réfléchir aux possibilités de publier un "tableau de pointage " pour transmettre à divers publics un message convaincant sur les inégalités sur le plan de la santé. C'est la dernière en date des retouches jugées nécessaires à l'évaluation des besoins en santé de la population : l'ajout de consultations systématiques et la prise de conscience de l'utilité d'un processus de consultation, tant pour la défense des droits que pour la réaffectation des ressources. D'autres articles dans ce numéro nous proposent des indicateurs qui étendent la portée et le rôle de la santé publique.

Par exemple, Schellenberg et ses collègues (page 369) partagent avec nous une étude révélatrice de l'écart entre les connaissances générales, les comportements et les méthodes de santé publique recommandées. Comme nous le montrent Yiannakoulias et coll. (page 374), les facteurs communautaires sont de puissants prédicteurs de la santé de la population. On trouvera aussi dans ce numéro des études nuancées et bien menées sur la santé de populations vulnérables. Dallaire et coll. (page 362) révèlent l'impact de certaines infections de l'enfance dans les communautés inuites isolées. Podymow et coll. (page 379) ont fait appel à des dossiers médicaux informatisés pour analyser certains états de santé chez les hommes sans abri à Ottawa. En comparant les données de deux provinces, Fischer et coll. (page 384) ont dégagé des tendances dans les décès prématurés associés aux surdoses. Une étude éclairante de Greaves et coll. (page 388) illustre méticuleusement les besoins non satisfaits des femmes marginalisées. Et dans un article important, Sloka et coll. montrent l'impact des programmes de supplémentation en acide folique dans la population de TerreNeuve-et-Labrador (page 393). Les enfants à risque de devenir obèses font l'objet d'un article de Galloway (page 353).

Ce numéro présente aussi une critique constructive des principes et des usages en santé publique. Kenny et coll. (page 402) font valoir la nécessité d'asseoir la santé publique sur des bases morales solides. Wen-kui et coll. (page 398) décrivent pour nous le cadre multiniveau qui optimise les interventions d'urgence en santé publique au Guangxi, une région de la Chine qui compte près de 50 millions d'habitants. Les définitions de la santé et de l'invalidité sont le reflet de postulats et de priorités de santé publique très profondément ancrés, comme le montre Wolbring (page 405). Muldoon et coll. font une critique de l'usage contemporain des expressions " soins primaires " et " soins de santé primaires " (page 409), tandis que Knaak (page 412) pose d'importantes questions à propos du discours sur l'allaitement maternel. Et O'Reilly et coll. jettent un nouvel éclairage sur le processus de la recherche interdisciplinaire (page 415).

Quand vous aurez fini votre lecture, revenez quelques instants à notre page couverture. Imaginez un " tableau de pointage " dont les données, exactes et complètes, imposeraient une nouvelle ligne d'action en santé publique...

Jeanette Ward, MBBS, MHPEd, Ph.D., FAFPHM

Rédactrice-réviseure scientifique intérimaire de la RCSP 